\title{
FAIRNESS DYNAMICS IN MULTIMEDIA COLLUDERS' SOCIAL NETWORKS
}

\author{
W. Sabrina Lin*, H. Vicky Zhao ${ }^{\dagger}$ and K. J. Ray Liu* \\ * ECE Dept., University of Maryland, College Park, MD 20742 USA \\ $\dagger$ ECE Dept., University of Alberta, Edmonton, AB T6G 2V4 Canada
}

\begin{abstract}
Multimedia social network analysis is a research area with growing importance, in which the social network members share multimedia contents with all different purposes and analyzing their behavior help design more secured and efficient multimedia and networking systems. In this paper, we focus on multimedia fingerprinting social network, in which multi-user collusion being a powerful attack, where a group of attackers collectively undermine the traitor tracing capability. During collusion, different colluders have different objective,s thus, the colluders form a social network and an how to achieve agreement on distributing the risk/profit among colluders and ensure fairness of the attack is a crucial question. This paper models the dynamics among colluders as a non-cooperative game, propose a general model of utility functions and study four different bargaining solutions of this game.
\end{abstract}

Index Terms- Multimedia social network analysis, game theory

\section{INTRODUCTION}

A social network is a social structure made of nodes (which are generally individuals or organizations) that are tied by one or more specific types of interdependency, such as values, friendship, conflict, financial exchange, trade, etc. In the paste decade, social network analysis has become a popular topic in sociology, economics, information science and many other disciplines in which people are studying how to model the relationships between members at all scales, from interpersonal to international.

In a multimedia social network, a group of members form a dynamically changing network infrastructure to share and exchange multimedia contents, as well as other resources. By participating in multimedia social networks, the members receive rewards by being able to access extra resources from other members, and they also contribute their own resources. Members in multimedia social networks aim to maximize their own payoff, and different users have different (and often conflicting) objectives. Thus, an important issue in multimedia social networks is to understand the strategies that members will play when negotiating with each other and study

The authors can be reached at wylin@eng.umd.edu, vzhao@ece.ualberta.ca, and kjrliu@eng.umd.edu. how they achieve fairness. Game theory $[1,2]$ provides a fundamental tool to study the fairness dynamics among multimedia social network members. By analyzing the human behavior in multimedia social networks, both the users and the system designer will have a clear picture of what's the profit every user can get in this multimedia social network, thus ultimately lead to systems with more secure, efficient and personalized services.

In this paper, without loss of generality, we use the multimedia fingerprinting system to illustrate the modelling and analysis of user behavior in multimedia social networks. Digital fingerprinting embeds a unique label, known as fingerprint, into every distributed copy to track the usage of multimedia data to protect multimedia from illegal usage and unauthorized redistribution. Multi-user collusion is a powerful attack in digital fingerprinting system, where a group of attackers work together to effectively remove the identifying information, thus these colluders form a social network.

In the colluders' social network, members collaborate with each other to reduce their chance of being caught and share the profit of redistributing the colluded redistributed multimedia content. Every member wishes to minimize his/her risk and maximize his/her profit, which definitely conflict with each other. To address this conflict, members in the colluders' social network have to agree on how to distribute the risk and achieve "fairness" of the attack. To analyze the dynamics among the members in colluders' social network, we model the members behavior as a non-cooperative game where each colluder tries to maximize his/her individual payoff under the fairness constraint. We consider different definitions of "fairness", investigate how the colluders would like to share the risk and the profit, and study different bargaining solutions: Nash-Bargaining, Max-Min, and Max-Sum solution.

The rest of the paper is as follows. Section 2 introduces the multimedia fingerprinting systems that we consider in this paper, and formulates the fairness dynamics among colluders. We define the utility functions and the four fairness criteria in Section 3. We show simulation results in Section 4, and conclusions are drawn in Section 5.

\subsection{Scalable Video Coding Systems}

Nowadays, scalable video coding is widely adopted to accommodate heterogenous networks and devices with different 
storage and computing capability. It decomposes the video sequence into different layers of different priority. The base layer contains the most important information of the video and is received by all users, and the enhancement layers gradually refine the reconstructed sequence at the decoder's side and are only received by users with sufficient bandwidth. Without loss of generality, we consider a two-layer temporally scalable video coding system, where different frames are encoded at different layers [3]. Take MPEG-2 video coding as an example, the base layer includes all the I frames, and the enhancement layer may contain all the $\mathrm{P}$ and $\mathrm{B}$ frames.

Define $F_{b}$ and $F_{e}$ as the sets containing the indices of the frames that are encoded in the base layer and the enhancement layer, respectively; and let $F^{(i)}$ be the set that contains the indices of the frames that user $\mathbf{u}^{(i)}$ receives. $U^{b}$ is the subgroup of users who receive the base layer only; and $U^{b, e}$ contains all users who subscribe to the high quality version containing both layers.

\subsection{Scalable Multimedia Fingerprinting System}

Fingerprint Embedding We use the spread spectrum embedding [4] to embed fingerprints in the host signal. Let $\mathbf{S}_{j}$ be the $j$ th frame in the video, and for each user $\mathbf{u}^{(i)}$ who subscribes to frame $j$, the content owner generates a unique fingerprint $\mathbf{W}_{j}^{(i)}$, with the same length as $\mathbf{S}_{j}$. The fingerprinted frame is $\mathbf{X}_{j}^{(i)}=\mathbf{S}_{j}+J N D_{j} \mathbf{W}_{j}^{(i)}$, which is distributed to $\mathbf{u}^{(i)}$. $J N D$ [4] here is used to control the energy of the embedded fingerprints and make the fingerprinted copy be perceptually the same as the original one. In this paper, we first generate independent vector from Gaussian distribution $\mathcal{N}\left(0, \sigma_{w}^{2}\right)$, and then apply Gram-Schmidt orthogonalization to generate orthogonal fingerprints for different users.

Multi-user Collusion In this paper, we only consider averaging based collusion because nonlinear collusion can be modelled as averaging collusion with additive noise, and all collusion attacks have similar performance with colluded copies of the same quality. During collusion, depending on the resolutions of their received copies, the colluders are divided into two non-overlapping subgroups. $S C^{b}$ is the set including the indices of the colluders who receive the base layer only and $S C^{b, e}$ contains the indices of the colluders who subscribe to the high quality version. $K^{b}$ and $K^{b, e}$ are the number of colluders in $S C^{b}$ and $S C^{b, e}$, respectively, and $K=K^{b}+K^{b, e}$ is the total number of colluders.

Without loss of generality, we consider the scenario where colluders who receive fingerprinted copies of the same resolution agree to share the same risk. Following the work in [3], colluders apply intra-group collusion first: for each frame $j \in F_{b}$ that they receive, colluders in $S C^{b}$ generate $\mathbf{Z}_{j}^{b}=$ $\sum_{k \in S C^{b}} \mathbf{X}_{j}^{(k)} / K^{b}$, and for each received frame $j \in F_{b} \cup F_{e}$, colluders in $S C^{b, e}$ calculate $\mathbf{Z}_{j}^{b, e}=\sum_{k \in S C^{b, e}} \mathbf{X}_{j}^{(k)} / K^{b, e}$. Then, the colluders apply inter-group collusion: for each frame $j \in F_{b}$ in the base layer, colluders generate $\mathbf{V}_{j}=\beta \mathbf{Z}_{j}^{b}+(1-$ $\beta) \mathbf{Z}_{j}^{b, e}+\mathbf{n}_{j}$ where $0 \leq \beta \leq 1$; and for each frame $j \in F_{e}$ in the enhancement layer, $\mathbf{V}_{j}=\mathbf{Z}_{j}^{b, e}+\mathbf{n}_{j} \cdot \mathbf{n}_{j}$ is the additive noise to further deter the detection performance.

Fingerprint Detection: When identifying colluders, the fingerprint detector first extracts the fingerprint $\mathbf{Y}_{j}$ from frame $j$ in the colluded copy. Then, for each user $\mathbf{u}^{(i)}$, the fingerprint detector calculates the detection statistics

$$
T N^{(i)}\left(\breve{F}^{(i)}\right)=\left(\sum_{j \in \breve{F}^{(i)}}\left\langle\mathbf{Y}_{j}, \mathbf{W}_{j}^{(i)}\right\rangle\right) / \sqrt{\sum_{j \in \breve{F}^{(i)}}\left\|\mathbf{W}_{j}^{(i)}\right\|^{2}},
$$

compares with a threshold $h$, and outputs the estimated coll) luder set $\widehat{S C}=\left\{i: T N^{(i)}>h\right\}$. When identifying colluders, the fingerprint detector can use fingerprints extracted from all layers collectively. The fingerprint detector can also examine each individual layer to determine whether a user is involved in collusion. For example, for user $i \in \mathbf{U}^{b, e}, \breve{F}^{(i)}$ has three choices, $F_{b} \cup F_{e}, F_{b}$ and $F_{e}$.

Different detection statistics have different means, and the one with the largest mean has the best detection performance. The work in [5] proposed to estimate the means of different detection statistics first, and then use the one with the largest estimated mean when identifying colluders. It was shown that information about the detection statistics' means helps significantly improve the detection performance; and the proposed self-probing fingerprint detector has approximately the same performance as the optimum one, which has perfect knowledge of the means and always select the detection statistics with the best performance.

\section{GAME MODEL OF HUMAN BEHAVIOR IN COLLUDERS' SOCIAL NETWORK}

In this section, we will first define the utility function of every member in the colluders' social network, find the feasible set of the game, and analyze possible bargaining solutions under different fairness criteria.

\subsection{Utility Function Definition}

During collusion, every member in the colluders' social network wants to minimize his/her own risk and maximizes his/her own profit. For colluder $\mathbf{u}^{(i)}$, his/her payoff function $\pi^{(i)}$ should be composed of two terms: colluder $i$ 's loss if being detected plus his/her profit as follows:

$$
\pi^{(i)}=-P_{d}^{(i)} * L^{(i)}+R^{(i)} .
$$

In (2), $P_{d}^{(i)}$ and $L^{(i)}$ stand for colluder $\mathbf{u}^{(i)}$ 's probability and loss of being detected, and $R^{(i)}$ is the profit that $\mathbf{u}^{(i)}$ gets by redistributing the colluded multimedia content. $L^{(i)}$ is the private information of every player in this game, and it is easy to prove that every player will claim his/her own lost being the maximum value that this game allow, thus, $L^{(i)}=$ $L^{(j)} \forall i, j \in S C$. Since the total profit of redistributing the colluded copy is proportional to its quality: the better the quality is, the more total profit the colluders can get, and in 
temporal scalable video coding scenario, video quality is proportional to the number of frames, thus we propose a general model of $R^{(i)}$ :

$$
R^{(i)}=\frac{F^{c} / F^{\max }}{\left[\sum_{j=1}^{K}\left(F^{(j)}\right)^{\gamma} D\left(P_{d}^{(j)}\right)\right] / M}\left(F^{(i)}\right)^{\gamma} D\left(P_{d}^{(i)}\right) .
$$

Where $F^{c}$ is the number of frames in the final colluded copy, $F^{\text {max }}$ is the largest number of frames among all the subscribers' copies, $F^{(i)}$ is the number of frames in $\mathbf{u}^{(i)}$ 's copy; $\mathrm{K}$ is the total number of colluders, $\mathrm{M}$ is the total number of subscribers, and $D(\bullet)$ is a non-decreasing function. Thus $F^{c}$ term illustrates the total profit of all the members in colluders' social network, which is shared by total number of colluders $K$, where $F^{\max }$ and $M$ are the normalization terms. Furthermore, profit is not often shared equally by all the members in colluders' social network; one simple reason is that colluders who subscribed to higher resolution copies are tended to ask more profit, since they already paid more money to get the higher resolution copies and if they quit the collusion, quality of the colluded copy will be lower and directly lead to lower total profit. $\left(F^{(i)}\right)^{\gamma}$ in (3) allow the colluders to adjust the profit distribution based on individual copy's quality, where $\gamma \geq 0$ can be determined by the agreement of the colluders' social network to control how unequal the profit distribution is: colluders who subscribed to higher resolution copies get more profit with higher $\gamma$. The other reason of unequal profit distribution that usually happens in colluders' social network is that some colluders are willing to be the risk-taker and at the same time, get more profit. So in our profit model, we include the non-decreasing function $D\left(P_{d}^{(i)}\right)$ to illustrate this kind of human behavior.

In the following sections, to simplify the analysis and without loss of generality, we assume the colluders who receive the same quality copies agree to share the same probability of being detected as in Section 2.2. Thus colluders who receive the low-resolution copies act as a single player in the game and they have the same utility $\pi^{b}$, while while colluders who have the high-resolution copies act as a single player during the bargaining process and they have the same utility $\pi^{b, e}$.

\subsection{Fairness Criteria}

Depending on the definition of fairness and the objectives of collusion, colluders select different collusion strategies and aim to reach agreement under different fairness criteria. In this section, we demonstrate the behavior analysis of colluders' social network by four commonly used fairness criteria during bargaining.

Absolute Fairness: The most straight-forward fairness criterion is absolute fairness, which means the utility of every member in the colluders' social network is equal, where

$$
\pi_{\text {Absolute }}=\pi^{(i)}=\pi^{(j)} \quad \forall i, j \in S C,
$$

and, since we assume colluders who receive the same quality copies have equal utility, (4) can be simplified to

$$
\pi_{\text {Absolute }}=\pi^{b}=\pi^{b, e}
$$

MaxMin Fairness: To guarantee the utility of every one who participate the colluders' social network, colluders can also select the collusion parameters to maximize the minimum utility over all members in the social network, that is,

$$
\pi_{\operatorname{maxmin}}=\max _{\beta} \min _{i}\left\{\pi^{(i)}: i \in S C\right\},
$$

which can also be simplified to

$$
\pi_{\text {maxmin }}=\max _{\beta} \min \left\{\pi^{b}, \pi^{b, e}\right\} \text {. }
$$

Max Sum Fairness: Under some circumstances, all the members in the colluders' social network have the same goal so that they are willing to maximize the total utility over the whole social network as follows:

$$
\pi_{\text {maxsum }}=\max _{\beta} \sum_{i \in S C} \pi^{(i)} .
$$

Max sum solution has a desired property that if it is feasible, it is Pareto-Optimal.

Proof: If $\pi_{\text {maxsum }}=K^{b} \pi_{\text {maxsum }}^{b}+K^{b, e} \pi_{\text {maxsum }}^{b, e}$ is feasible but not Pareto-Optimal, then there exists $\left(\pi_{\text {maxsum }}^{b}, \pi^{b, e^{\prime}}\right)$ or $\left(\pi^{b^{\prime}}, \pi_{\text {maxsum }}^{b, e}\right)$ in feasible set where $\pi^{b^{\prime}}>\pi_{\operatorname{maxsum}}^{b}, \pi^{b, e^{\prime}}>$ $\pi_{\text {maxsum }}^{b, e}$ by the definition of Pareto-Optimal. Thus there exists a feasible $\pi^{\prime}>\pi_{\text {maxsum }}$, which contradict the definition in (8).

Nash-Bargaining Solution: Nash-Bargaining solution, which is also Pareto-Optimal [1], is a famous bargaining solution in game theory, in which the basic idea being proportional fairness. Definition of general Nash-Bargaining solution is as follows:

$$
\begin{aligned}
g\left(\pi^{b}, \pi^{b e}\right) & =\left(\pi^{b}-\pi^{b *}\right)^{B_{b}}\left(\pi^{b e}-\pi^{b e *}\right)^{B_{b, e}} \\
\text { where } \pi^{b *} & =\min _{\beta}\left\{\pi^{b}\right\} \quad, \quad \pi^{b e *}=\min _{\beta}\left\{\pi^{b e}\right\}
\end{aligned}
$$

and $B_{b}, B_{b, e}$ are the bargaining powers of $S C^{b}, S C^{b, e}$, respectively. When $B_{b}=B_{b, e}=1$, Nash-Bargaining solution divides the additional utility between the two players in a ratio that is equal to the rate at which this utility can be transferred. If $B_{b} \neq B_{b, e}$, then the bargaining solution deviates from the proportional fairness solution and favors the player with higher bargain power.

\section{CASE STUDY AND SIMULATION RESULTS}

In this section, we take two different utility functions as examples to illustrate the human behavior dynamics of colluders' social network. To have a clear picture of the agreement that the four fairness criteria will achieve, we first use a simple utility function as follows:

$$
\pi^{(i)}=-P_{d}^{(i)} * L+\frac{F^{c} / F^{\max }}{K / M},
$$

which is a special case of (3) where $\gamma=0$ and $D\left(P_{d}^{(i)}\right)=1$, meaning the profit of redistributing the colluded copy is equally distributed to all the colluders. In this case, the feasible region is convex, and the Pareto-Optimal set also exists as proofed in our previous work [6]. In real-world social networks, profit is usually distributed unequally because every member has different personal concern and position in the society, thus we also consider the more general utility function, 


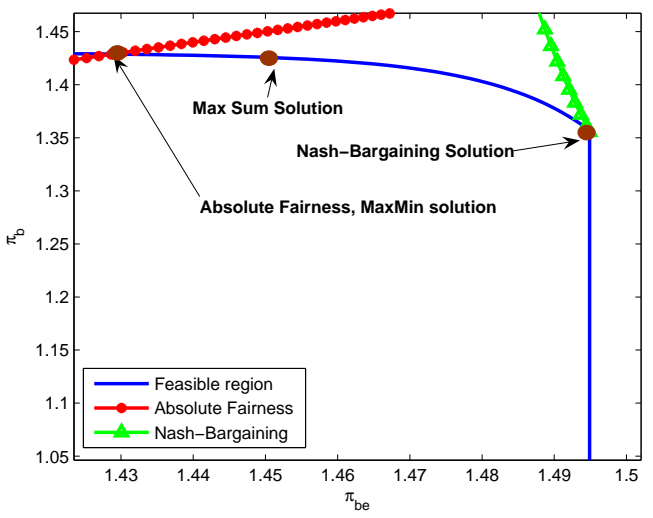

Fig. 1. Feasible Region and Bargaining Solutions with utility function as in (10), $P_{f a}=10^{-3}, N_{b}=N_{e}=50000, K^{b}=$ $80, K^{b, e}=170$, and $\left|U^{b}\right|=\left|U^{b, e}\right|=250$.

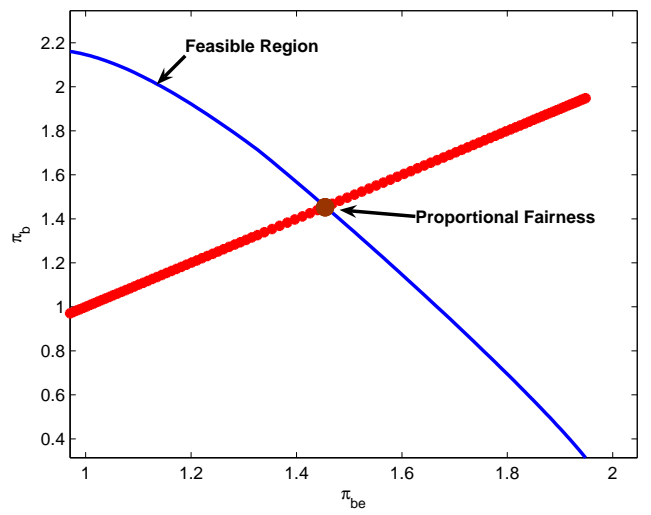

Fig. 2. Feasible Region and Proportional Fairness Solutions with utility function as in (11), $P_{f a}=10^{-3}, N_{b}=N_{e}=$ 50000, $K^{b}=80, K^{b, e}=170$, and $\left|U^{b}\right|=\left|U^{b, e}\right|=250$.

$$
\begin{aligned}
& \pi^{(i)}=-P_{d}^{(i)} * L+ \\
& \quad \frac{F^{c} / F^{\max }}{\left(K^{b}\left(F^{b}\right)^{0.1}+K^{b, e}\left(F^{b, e}\right)^{0.1}\right) / M}\left(F^{(i)}\right)^{0.1} P_{d}^{(i)}(1)
\end{aligned}
$$

to illustrate the feasible region when the colluders distribute profit proportional to each copy's quality and risk (probability of being detected).

\subsection{Simulation Setting and Results}

In our simulations, we first generate independent vectors following Gaussian distribution $\mathcal{N}(0,1)$, and then apply GramSchmidt orthogonalization to generate orthogonal fingerprints. The lengths of the fingerprints embedded in the base layer and the enhancement layer are $N_{b}=N_{e}=50000$, and both two layers contain 20 frames, respectively. The total number of users is 500, where $U^{b}=U^{b, e}$. The probability of accusing an innocent user, $P_{f a}$, is $10^{-3}$. Among the $K=250$ colluders, $K^{b}=80$ of them receive the fingerprinted base layer only, and the other $K^{b, e}=170$ of the colluders receive fingerprinted copies of high resolution.

Figure 1 shows the feasible region and the four bargain- ing solutions in Section 3.2 with utility function as in (10), and bargaining power in (9) $B_{b}=1, B_{b, e}=3$. In this game, since utility of one player is a non-decreasing function of the other player's utility, it can be easily proofed that if absolute fairness solution is feasible, then it is also a max-min solution. Compared to the absolute fairness solution, the max-sum solution gives the group with more people more utility, which is, $S C^{b, e}$ in our case. The Nash-Bargaining with bargain power $B_{b}=1, B_{b, e}=3$ even more favor $S C^{b, e}$ since $B_{b, e}>B_{b}$, which usually happens in real-world social network: colluders with higher resolution copies have more bargain power.

Figure 2 shows the feasible region, and, since our analysis in on the bargaining level, the trend of the bargaining solutions are independent of utility function definition, which means our methodology can fit to different problems once the utility function is defined. Thus here we only show the "absolute fairness solution" under proportional profit distribution, which also has proportional fairness characteristics, thus is labelled as "proportional fairness" in Figure 2. Comparing Figure 1 and Figure 2, it is clear that the maximum utility that $S C^{b}$ can achieve is higher if profit is distributed proportionally, because $K^{b}<K^{b, e}$, the highest risk of $S C^{b}$ is higher then $S C^{b, e}$.

\section{CONCLUSIONS}

This paper studies the behavior modelling and analysis of the dynamics in colluders' social network to achieve different fairness of collusion. We model the fairness dynamics among colluders as a non-cooperative game, where each colluder aims to maximize his/her own utility through bargaining to achieve fair agreement. We propose a general model of utility functions which allows unequal profit-distribution, and analyze human behavior by four bargaining criteria: absolute fairness, max-min, max-sum, and Nash-Bargaining solution. Our analysis shows that in colluders' social network, colluders choose different points in the feasible set, depending on the colluders' definition of "fairness" and their agreement on how to distribute the risk and the profit among themselves, and our methodology can fit human behavior analysis in different social networks.

\section{REFERENCES}

[1] G. Owen, Game Theory, Academic Press, 3rd edition, 1995.

[2] D. Fudenberg and J. Tirole, Game Theory, MIT Press, 1991.

[3] H. Zhao and K. J. R. Liu, "Behavior forensics for scalable multiuser collusion: fairness versus effectiveness," IEEE Tran. on Information Forensics and Security, vol. 1, no. 3, pp. 311-329, Sept. 2006.

[4] C. Podilchuk and W. Zeng, "Image adaptive watermarking using visual models," IEEE Journal on Sel. Area in Comm., vol. 16, no. 4, pp. 525540, May 1998.

[5] W. S. Lin, H. V. Zhao, and K. J. R. Liu, "Scalable multimedia fingerprinting forensics with side information," IEEE Int. Conf. on Image Processing, pp. 2293-2296, Oct. 2006.

[6] W. S. Lin, H. V. Zhao, and K. J. R. Liu, "Multi-user collusion behavior forensics: game-theoretic formulation of fairness dynamics," IEEE Int. Conf. on Image Processing, Sept. 2007. 